\title{
SARS-CoV-2 in a Pediatric Patient Requiring Mechanical Ventilation and Multi-Drug Therapy
}

\author{
Elliott S. Gordon ${ }^{\mathrm{a}, \mathrm{c}}$, Michael B. Dinerman ${ }^{\mathrm{b}}$
}

\begin{abstract}
Severe acute respiratory syndrome coronavirus 2 (SARS-CoV-2) is a novel viral pathogen that was found to disproportionately affect older adults, often sparing pediatric patients from moderate to severe illness. We report on a case of a 12-year-old patient admitted through the emergency department with bilateral pneumonia, thrombocytopenia and hematuria, who subsequently developed respiratory failure requiring intubation and mechanical ventilation. The patient was found to be SARS-CoV-2-positive. The patient's management strategy is discussed.
\end{abstract}

Keywords: Coronavirus; COVID-19; SARS-CoV-2; Pediatrics; Intubation; Mechanical ventilation

\section{Introduction}

Novel coronavirus disease 2019 (COVID-19), medically referred to as severe acute respiratory syndrome coronavirus 2 (SARS-CoV-2), is a zoonotic viral illness, first identified in late December 2019 in Wuhan, China [1, 2]. Though exposure to wildlife accounts for less than $2 \%$ of documented cases, bats are initially believed to have been the host reservoir; however, direct person-to-person transmission has emerged as a more rapid transmission route [3]. The illness rapidly spreads between close contacts, presumably via aerosolized respiratory secretions, with precipitous spread from Asia to Europe and the Americas in rapid succession [1,4]. Many patients present with several days of fever and dry cough, symptoms attributed to influenza or other respiratory pathogens [3]. Some patients present with abdominal pain, nausea, vomiting or diarrhea [5].

Manuscript submitted April 15, 2020, accepted May 2, 2020

Published online June 18, 2020

aDepartment of Pediatrics, Mercer University School of Medicine, 777 Hemlock Street, MSC-42, Macon, GA, 31201, USA

${ }^{b}$ Children's Healthcare of Atlanta, Scottish Rite Hospital, 1001 Johnson Ferry Road NE, Atlanta, GA 30342, USA

${ }^{\mathrm{c} C o r r e s p o n d i n g ~ A u t h o r: ~ E l l i o t t ~ S . ~ G o r d o n, ~ D e p a r t m e n t ~ o f ~ P e d i a t r i c s, ~ M e r c e r ~}$ University School of Medicine, 777 Hemlock Street, MSC-42, Macon, GA 31201, USA. Email: gordon.elliott@navicenthealth.org

doi: https://doi.org/10.14740/ijcp370
A large-scale analysis of over 72,000 patient cases published in JAMA (February 2020) shows that less than $2 \%$ of cases occurred in patients under the age of 19 years, with fewer than $5 \%$ of cases deemed "severe" involving respiratory failure, septic shock, or multi-organ system failure [6].

We report a case involving a 12 -year-old patient who presented on three occasions to the emergency department (ED) at a large urban hospital with subjective fever at home for 5 days. To our knowledge, this patient represents one of the first pediatric patients to require intubation and intensive care management following a diagnosis of SARS-CoV-2-induced pneumonia with subsequent respiratory failure.

\section{Case Report}

A 12-year-old, previously healthy female of African descent presented with her mother to the ED at a large urban children's hospital. Mother reported that the patient's symptoms began with subjective fever, controlled with acetaminophen in earlyto-mid March. On day 2 of illness, after $36 \mathrm{~h}$ of fever to 39.6 ${ }^{\circ} \mathrm{C}$ (axillary), the patient was brought to the $\mathrm{ED}$. At that time, the patient was found to be febrile to $39.6{ }^{\circ} \mathrm{C}$ without any other abnormality noted on exam (Table 1).

The patient was diagnosed with a non-specific viral illness. She was noted to be taking oral liquids without a problem following oral ondansetron $(4 \mathrm{mg})$ administration. The patient stated that she "felt better" and agreed to symptomatic management with ondansetron, acetaminophen and oral fluid replacement at home.

On day 4 of illness, the patient returned to the ED with fever (maximum temperature at home: $106^{\circ} \mathrm{F}$ axillary), newonset non-productive cough and vomiting (Table 2).

At that time, a urinalysis obtained from a "dirty" catch demonstrated hematuria, mucus, bacteria and squamous epithelial cells without significant pyuria. Metabolic panel was within normal limits and rapid streptococcal testing was negative (Table 3). The patient received a normal saline bolus and was discharged home with recommendations for output primary care follow-up.

On her third visit to the ED, complaints consisted of persistent fever and emesis, with an absence of cough or difficulty breathing. At triage, vitals showed hypotension (blood pressure (BP) 90/71), pyrexia $\left(39.6{ }^{\circ} \mathrm{C}\right)$ and hypoxia $\left(\mathrm{SaO}_{2}\right.$ $89 \%$ on room air). Saturations improved to $92 \%$ on $2 \mathrm{~L}$ of oxygen by nasal cannula. Her respiratory rate ranged from 26 to 35 breaths per minute and she appeared intermittently 
Table 1. Emergency Department Vital Signs and Examinations Results for Visit \#1 Prior to Admission

\begin{tabular}{ll}
\hline Blood pressure (mm Hg) & - \\
Heart rate (beats per minute) & 139 \\
Respiratory rate (breaths per minute) & 20 \\
Temperature $\left({ }^{\circ} \mathrm{C}\right)$ & 39.6 \\
Pulse Ox $(\%)$ & $100 \%$ on room air \\
Weight $(\mathrm{kg})$ & 61 \\
Constitutional & Active, no acute distress, alert, well hydrated \\
Head/ears/nose/throat & Bilateral tympanic membranes are normal without erythema or bulging. Oral mucosa is moist. \\
Eyes & Conjunctivae normal. Pupils are equal, round and reactive to light. \\
Neck & Musculoskeletal: normal range of motion and neck supple. \\
Cardiovascular & Rate and rhythm: normal rate and regular rhythm. Heart sounds: S1 normal and S2 normal. \\
Pulmonary & $\begin{array}{l}\text { Pulmonary effort is normal. No respiratory distress or retractions. Normal breath sounds and air entry. } \\
\text { No decreased air movement. No wheezing. }\end{array}$ \\
Abdominal & Bowel sounds are normal. There is no distension. Abdomen is soft and flat. There is no abdominal \\
& tenderness, guarding or rebound. No right lower quadrant tenderness. Able to hop, smiles while hopping. \\
\hline Integumentary & Skin is warm and dry. Capillary refill takes less than 2 s. No petechiae or rash, purpura or mottling. \\
Neurological & Patient is alert with no focal neurologic deficits. \\
\hline
\end{tabular}

Table 2. Emergency Department Vital Signs and Examinations Results for Visit \#2 Prior to Admission

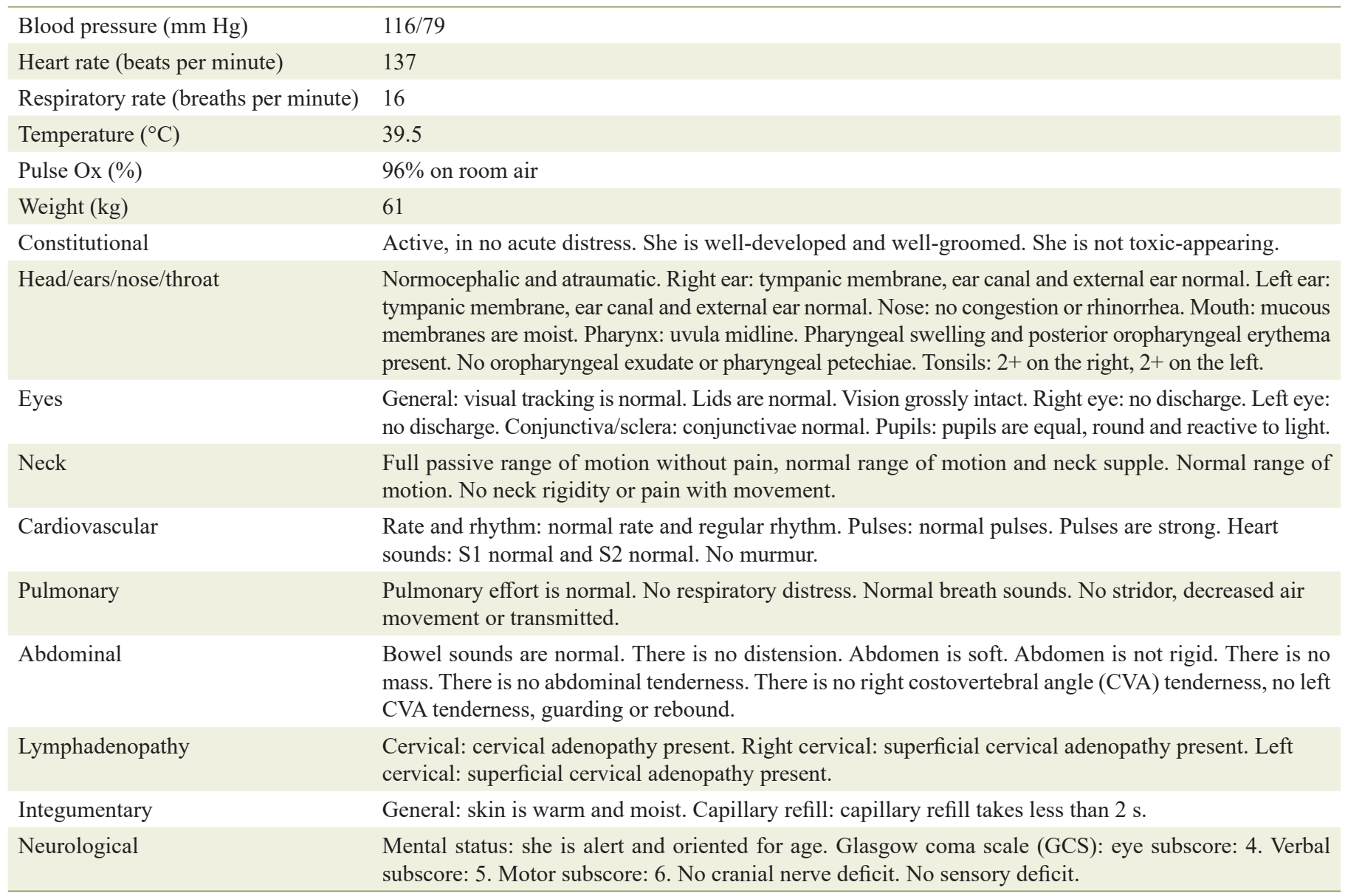


Table 3. Emergency Department Visit \#2 Laboratory Workup

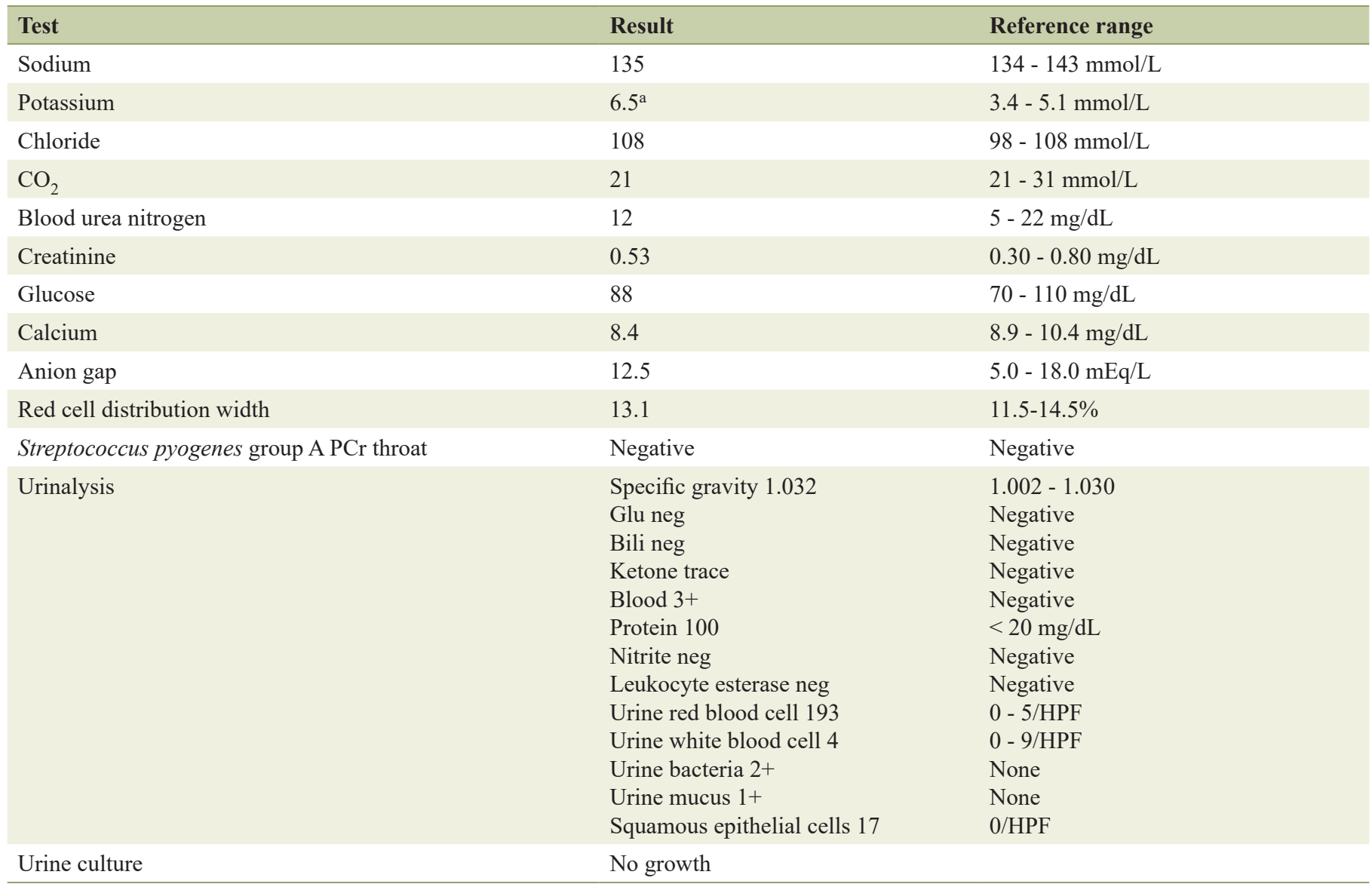

aGrossly hemolyzed.

Table 4. Emergency Department Vital Signs and Examinations Results for Visit \#3 Prior to Admission

\begin{tabular}{ll}
\hline Blood pressure (mm Hg) & $90 / 71$ \\
Heart rate (beats per minute) & 129 \\
Respiratory rate (breaths per minute) & $26-35$ \\
Temperature $\left({ }^{\circ} \mathrm{C}\right)$ & 39.6 \\
Pulse Ox (\%) & $89 \%$ on room air \\
Constitutional & Active. Not in acute distress. Non-toxic-appearing. Able to speak in full sentences. \\
Head/ears/nose/throat & $\begin{array}{l}\text { Normocephalic and atraumatic. Right and left external ear normal. No congestion or rhinorrhea. Mucous } \\
\text { membranes are moist. No oropharyngeal exudate or posterior oropharyngeal erythema. }\end{array}$ \\
\hline Eyes & $\begin{array}{l}\text { Right eye: no discharge. Left eye: no discharge. Extraocular movements intact. Conjunctiva/sclera: } \\
\text { conjunctivae normal. }\end{array}$ \\
\hline Neck & Normal range of motion and neck supple. \\
Cardiovascular & Regular rhythm. Tachycardia present. Pulses: normal pulses. Normal heart sounds. \\
Pulmonary & Tachypnea and respiratory distress present. No nasal flaring or retractions. \\
Abdominal & Abdomen is flat. Bowel sounds are normal. There is no distension or tenderness to palpation. \\
\hline Lymphadenopathy & No cervical lymphadenopathy appreciated on exam. \\
Integumentary & Skin is warm and dry. Capillary refill takes $2-3 \mathrm{~s}$. \\
Neurological & No focal deficit present. She is alert and oriented $\times 4$. Mood \\
& normal. Thought content normal. Judgment normal. \\
\hline
\end{tabular}




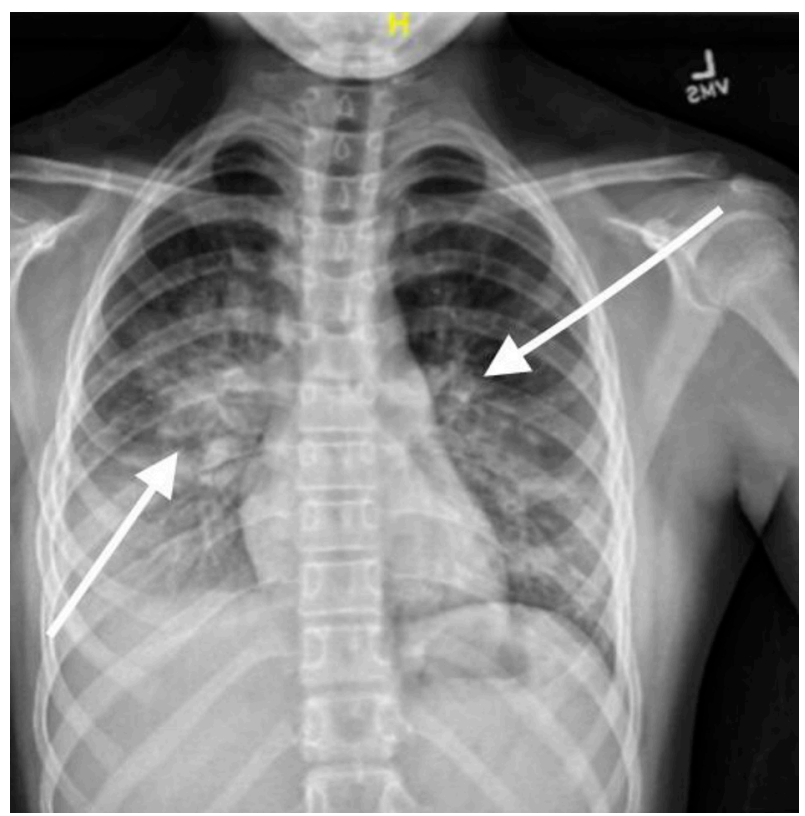

Figure 1. Chest $\mathrm{X}$-ray, anteroposterior (AP) view on third presentation to emergency department showing signs of pneumonia with consolidation.

uncomfortable, though non-toxic. She was started on heated high flow oxygen, $10 \mathrm{~L} / \mathrm{FiO}_{2} 35 \%$ and felt "more comfortable" (Table 4).

There was no history of recent travel in the 21 days preceding admission, sick contacts, or contacts with individuals with recent travel history or known COVID-19 exposures or diagnoses. However, the patient lives in a large urban center with known cases of COVID-19 and presented for evaluation following the closure of her county due to an outbreak of cases. Vaccinations were up to date, including against influenza A and B in August 2020. Of note, the patient's mother, father and 13 -year-old brother are all healthy and developmentally appropriate, with no significant past medical history. Family history is significant for patient's maternal grandfather with medical history of diabetes mellitus.

Radiographic imaging revealed significant bilateral parahilar and basilar airspace opacities (right greater than left) consistent with pneumonia and atelectasis, as well as a right pleural effusion (Figs. 1, 2).

The patient's initial workup included a complete blood count with differential, blood culture, complete metabolic panel, inflammatory markers, respiratory viral panel, urinalysis and urine culture (Table 5).

Given the results showing a platelet count of 8 and a repeat count of 10, disseminated intravascular coagulation (DIC) panel, fibrinogen and ferritin were ordered. Initially, prolonged partial thromboplastin time (PTT) (49.5 (26 - $38 \mathrm{~s})$ ), elevated ferritin (481.05 (13.7 - $78.8 \mathrm{ng} / \mathrm{mL}))$, normal PT (15.1 (12.6 $15.9 \mathrm{~s}))$, elevated D-dimer $(432(0-220 \mathrm{ng} / \mathrm{mL}))$ and normal fibrinogen $(376(200-400 \mathrm{mg} / \mathrm{dL}))$ were observed. However, through the course of hospitalization, the patients PT, PTT, Ddimer and fibrinogen all became elevated, well above reference ranges for the hospital laboratory.

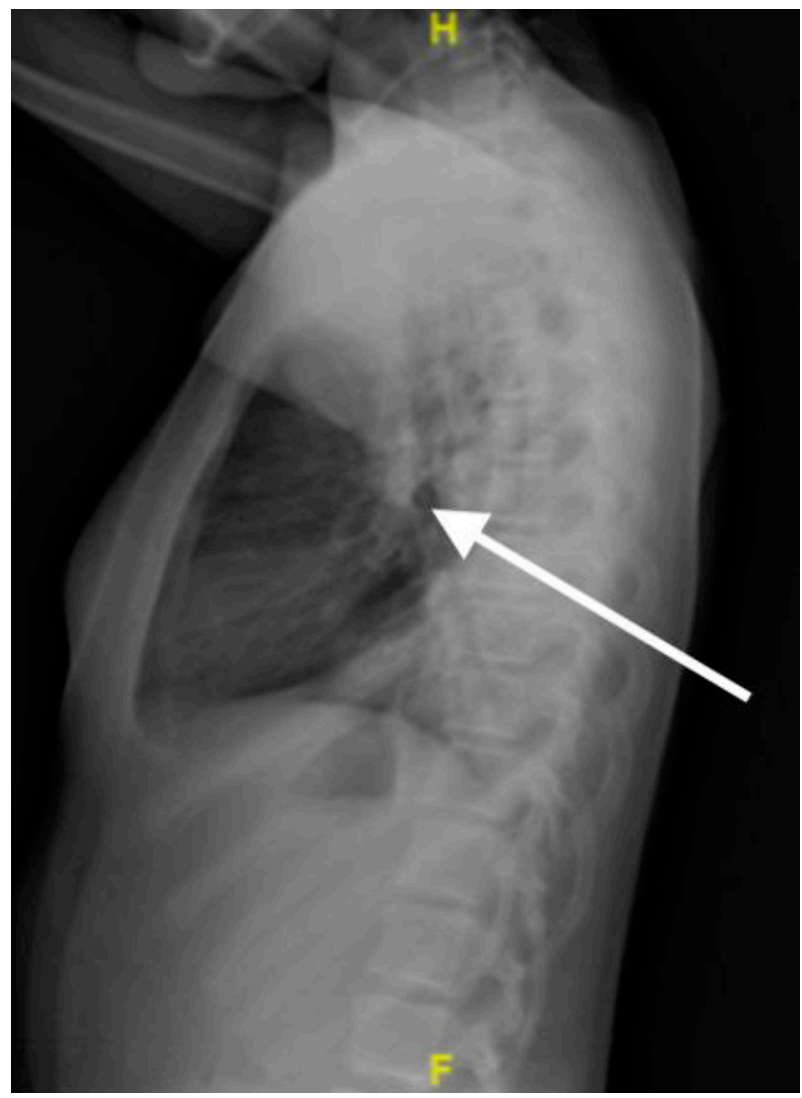

Figure 2. Chest X-ray, lateral view on third presentation to emergency department showing signs of pneumonia with areas of consolidation.

The patient received a normal saline bolus with improvement in her blood pressure. Ampicillin and ceftriaxone were administered. The patient was transferred in stable condition to the intensive care unit (ICU) for further care.

In the ICU, oxygen therapy was increased to $15 \mathrm{~L} / \mathrm{FiO}_{2}$ $100 \%$ and subsequently bilevel positive airway pressure (BiPAP). The patient's chest radiographs continued to show worsening signs of acute respiratory distress syndrome (ARDS). Viral testing was obtained.

Through hospital day 1 and overnight into hospital day 2, the patient continued to require increasing levels of respiratory support, with worsening clinical picture (Fig. 3) necessitating intubation with mechanical ventilation and nitric oxide therapy. Antibiotic therapy was switched to rocephin and vancomycin on hospital day 2 and subsequently cefepime, azithromycin and clindamycin, in various combinations (Table 6).

Multiple doses of intravenous immune globulin (IVIG; $1 \mathrm{~g} / \mathrm{kg} \mathrm{IV}$ ) were administered for severe thrombocytopenia, in addition to platelet transfusion, with minimal improvement. Solumedrol (100 mg IV for 1 day) was given in the treatment of presumptive immune thrombocytopenia. By hospital day 3, platelet count had improved to 34 and 151 by hospital day 8 . On hospital day 4 , immunologic workup was commenced, notable for elevated interleuken-6 (IL-6; $10(<5 \mathrm{pg} / \mathrm{mL}))$. 
Table 5. Emergency Department Visit \#3 Laboratory Workup

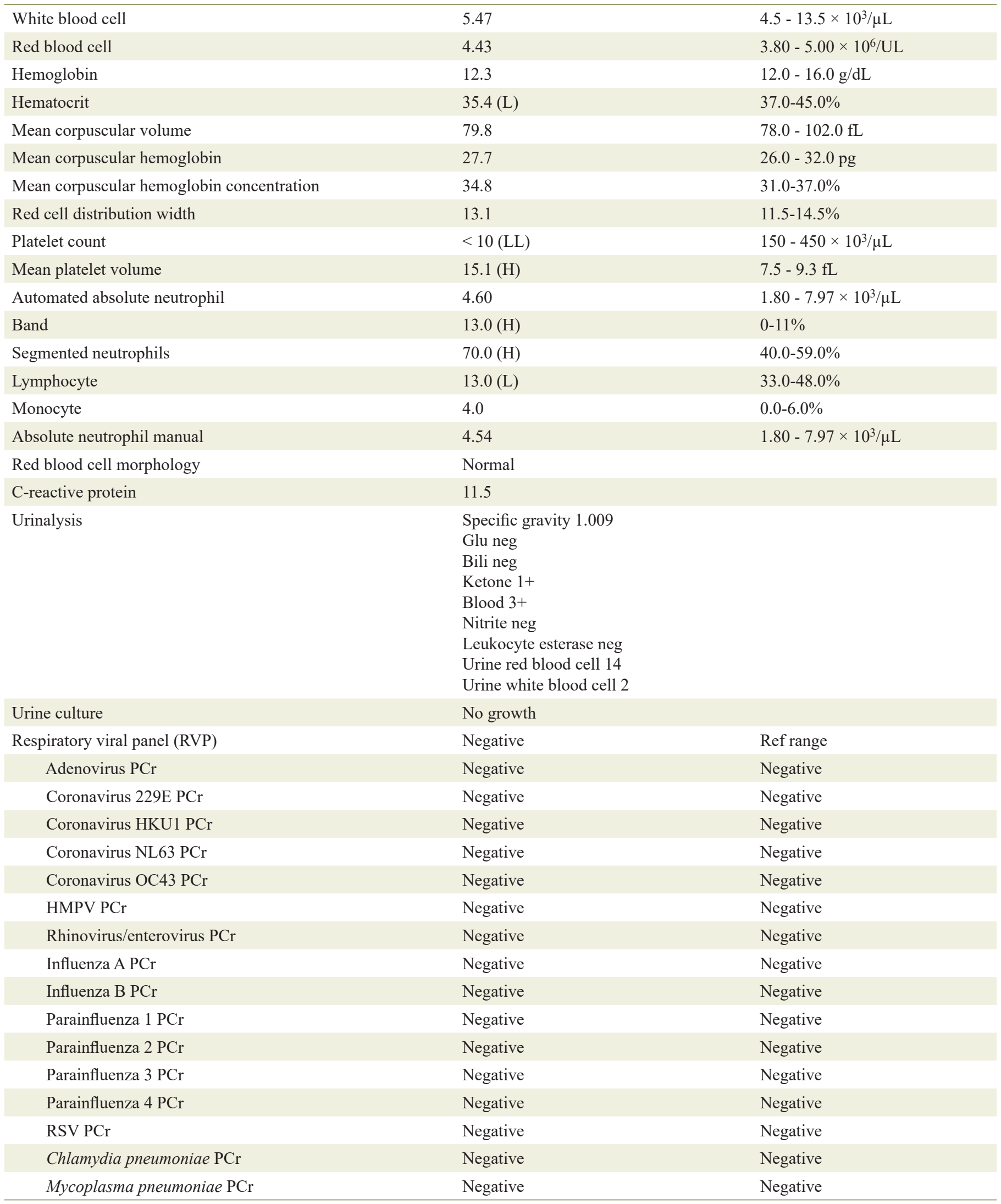




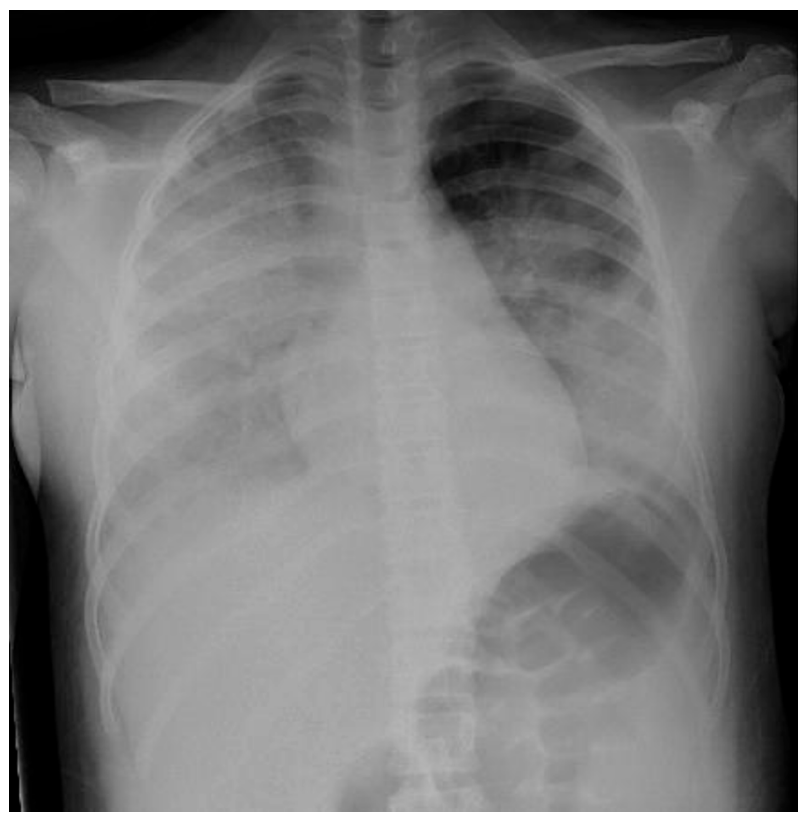

Figure 3. Chest $X$-ray, hospital day 1 showing signs of worsening acute respiratory distress syndrome (ARDS).

On hospital day 5, COVID-19 PCR was detected; the patient was SARS-CoV-2-positive. Given anecdotal reports of hydroxychloroquine in patients with COVID-19, this therapy was trialed on hospital days 6 through 8 , without signs of improvement. The patient was started on tocilizumab for its anticytokine effects, to dampen hyper-inflammation associated with sepsis, and due to emerging trials showing success in patients with ARDS due to COVID-19. On hospital day 7 (Fig. 4), approval for trial with remdesivir was obtained, which was started on hospital day 8 (Table 6; Fig. 5).

The patient's repeat chest radiograph showed signs of im-

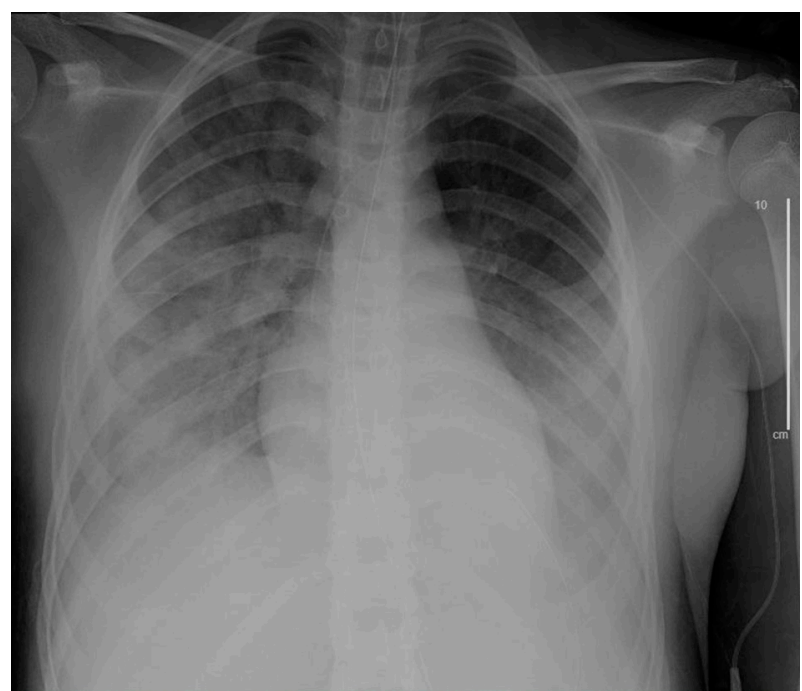

Figure 4. Chest $\mathrm{X}$-ray, anteroposterior (AP) view on the day prior to start of remdesivir trial (hospital day 7 ).

provement on hospital day 8 , with weaning of ventilator settings through hospital day 15 (Figs. 6-8).

The patient remained intubated on a ventilator for 13 days before extubation to high flow nasal cannula for 2 days and nasal cannula for 1 day. She then received 9 days of inpatient rehabilitation therapies due to global weakness, requiring extensive speech, physical and occupational therapy prior to discharge home.

\section{Discussion}

This previously healthy, 12-year-old female with no significant past medical history, presented with a 5-day history of fever

Table 6. Antibiotic, Antimicrobial and Antiviral Therapy Received During Hospitalization in Chronological Order as Medication Was Added to Treatment Plan

\begin{tabular}{llllll}
\hline Therapy & Dosage & Route of administration & Frequency & Duration of treatment & Days received \\
\hline Ceftriaxone & $2 \mathrm{~g}$ & IV & Daily & 7 days & Admission - HD 3 \\
\hline Ampicillin & $2 \mathrm{~g}$ & IV & Daily & 1 day & Admission - HD 1 \\
Albuterol & $2.5 \mathrm{~g}$ & Inhaled nebulizer therapy & Every $3 \mathrm{~h}$ PRN & 14 days & Admission - HD 17 \\
Vancomycin & $20 \mathrm{mg} / \mathrm{kg}$ & IV & Every $8 \mathrm{~h}$ & 5 days & HD $2-6$ \\
Cefepime & $2 \mathrm{~g}$ & IV & Every $8 \mathrm{~h}$ & 7 days & HD $3-10$ \\
Azithromycin & $10 \mathrm{mg} / \mathrm{kg}$ & IV & Daily & 2 days & HD $3-4$ \\
Azithromycin & $5 \mathrm{mg} / \mathrm{kg}$ & IV & Daily & 3 days & HD $5-7$ \\
Tocilizumab & $600 \mathrm{mg}$ & IV & Daily & 1 day & HD 7 \\
Tocilizumab & $600 \mathrm{mg}$ & IV & Every $12 \mathrm{~h}$ & 1 day & HD 8 \\
Hydroxychloroquine & $400 \mathrm{mg}$ & IV & Twice daily & 3 days & HD $6-8$ \\
Clindamycin & $10 \mathrm{mg} / \mathrm{kg}$ & IV & Every $8 \mathrm{~h}$ & 3 days & HD $7-9$ \\
Remdesivir & $200 \mathrm{mg}$ & IV & Daily & 1 day & HD 8 \\
Remdesivir & $100 \mathrm{mg}$ & IV & Daily & 9 day & HD $9-17$ \\
\hline
\end{tabular}




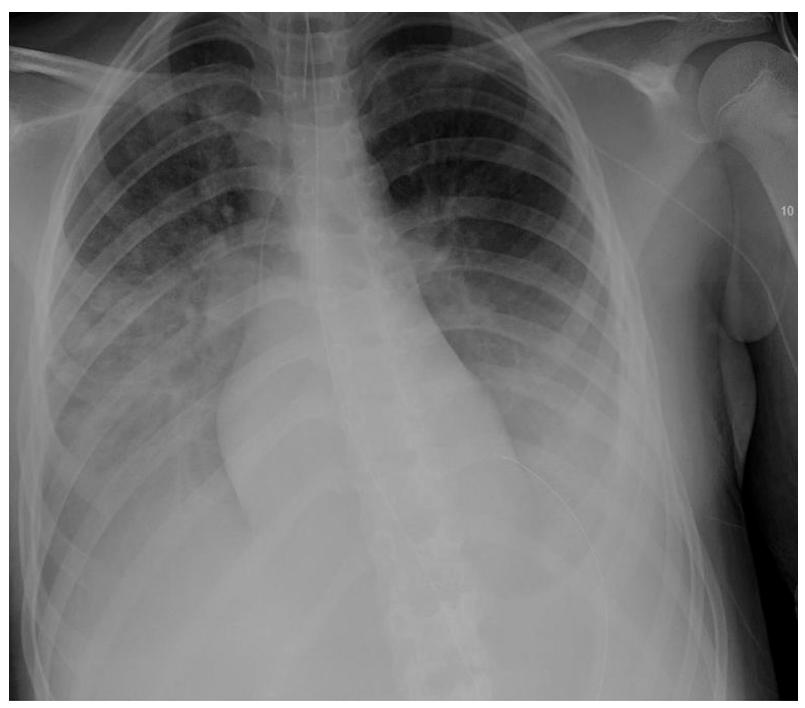

Figure 5. Chest X-ray, anteroposterior (AP) view on the day with remdesivir trial commenced (hospital day 8).

followed by abdominal pain, vomiting and increased work of breathing, found to have significant thrombocytopenia, bilateral pneumonia and respiratory failure as a result of COVID-19.

To our knowledge, this is the first pediatric patient in a large urban city in North America to fall critically ill due to this disease process, requiring mechanical ventilation, intensive care management and rehabilitation services prior to discharge home.

Recent reports suggest that less than $10 \%$ of children experience severe illness. When compared with adults, only one-third as many children have become critically ill $[1,7]$. Studies report that remarkably fewer children present acutely ill. Viral tests demonstrate that children may be asymptomatic carriers [1]. Virologists have hypothesized that the difference between adults and children stems from the fact that

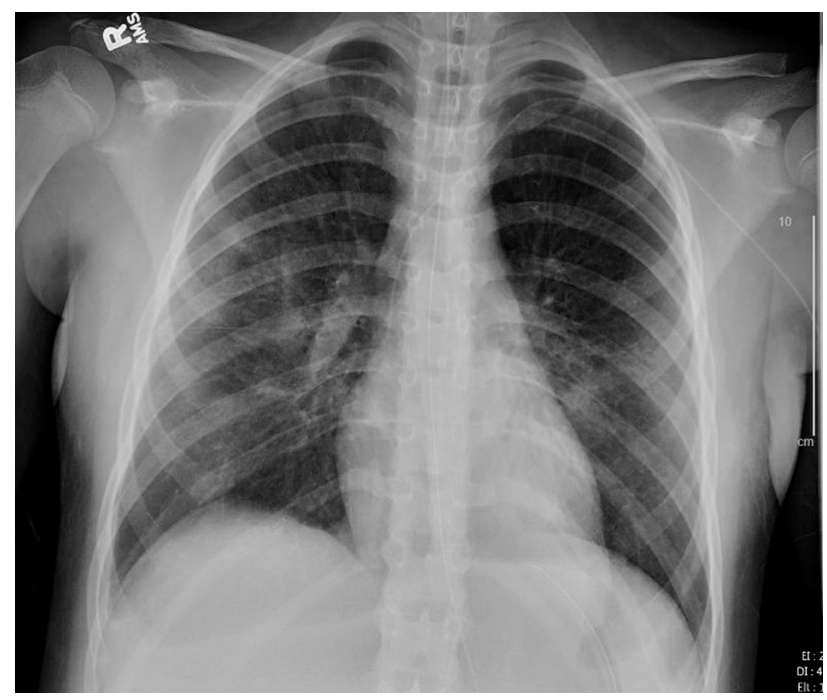

Figure 6. Chest $\mathrm{X}$-ray, anteroposterior (AP) view on the day following start of remdesivir (hospital day 9).

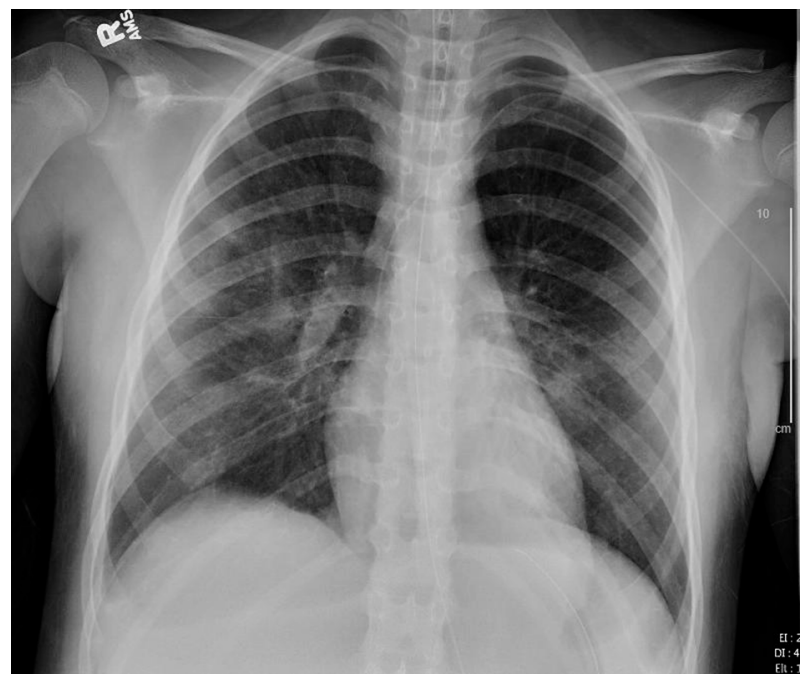

Figure 7. Chest $\mathrm{X}$-ray, anteroposterior (AP) view after 2 days of remdesivir therapy (hospital day 10).

an adult's immune system has been exposed to more pathogens and mounts a hyper-exaggerated inflammatory immune response which in turn may damage the host [8]. Similarly, hyper-inflammation mediated by significant cytokine release has been noted in patients who have COVID-19: "cytokine profile resembling (secondary $\mathrm{HLH}$ ) is associated with COVID-19 disease severity" [9]. It was noted that patients with significantly elevated IL-6 and ferritin carried a poor prognosis [9].

In our patient's case, identifiable host factors have been limited. With seemingly no past medical history, it is hypothesized that she may have been exposed to novel pathogens in her native country or more distant travels, a pathogen that may have been similar to SARS-CoV-2 or mimicking its effect, resulting in an exaggerated immune response.

Adult patients infected with SARS-CoV-2 frequently present with lymphopenia and mild thrombocytopenia with a platelet count rarely less than 100 [10]. Significant thrombocy-

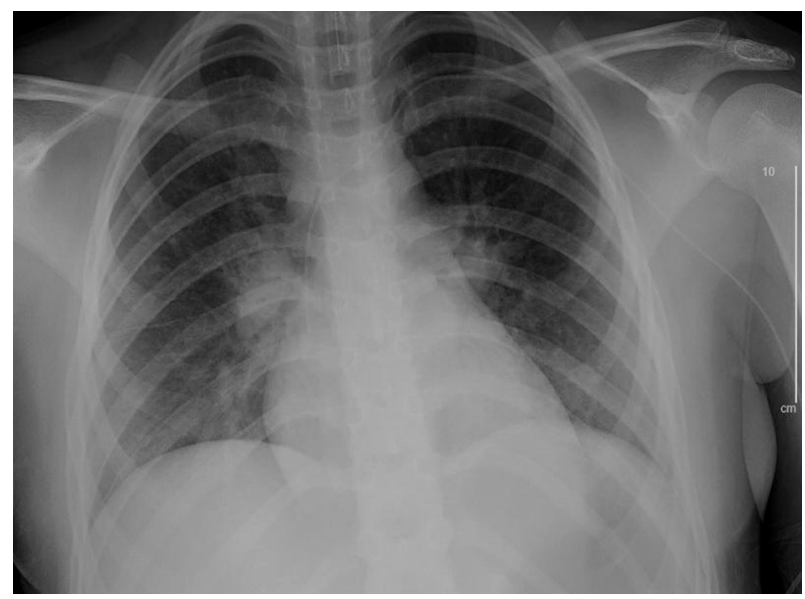

Figure 8. Chest $\mathrm{X}$-ray, anteroposterior (AP) view after 7 days of remdesivir therapy (hospital day 15). 
topenia has been deemed a worse prognostic indicator in these patients, denoting more severe disease and increased mortality $[10,11]$. Our patient was admitted after approximately 5 days of illness. It is likely that 5 - 10 days prior to presentation she contracted SARS-CoV-2 and was asymptomatic while the virus replicated and her body mounted a response. It is common for patients who are admitted to intensive care settings to present with thrombocytopenia as a direct result of platelet and complement activation or immune consumption $[12,13]$.

In general, patients with SARS-CoV-2 are found to have ground glass opacities in peripheral or basilar lung fields on chest radiographs. Disease severity has been associated with greater lung segment involvement [14]. Dense consolidation and pleural effusions are uncommon, with pleural effusion accounting for less than $1 \%$ of all computed tomography (CT) findings $[15,16]$. Additionally, bilateral lung involvement was seen in the vast majority of patients presenting in the late stages of disease, with consolidation clearing several weeks following illness [15]. In our patient's case, it is probable that she was asymptomatic with significant disease progression before presenting to the ED.

Hematuria has been described in the limited literature in older patients with COVID-19 with renal insufficiency and increased mortality [17]. Adult urine specimens often reveal asymptomatic microscopic hematuria with one systematic review of 80,000 patients, citing the prevalence as high as $31 \%$ of cases [18]. This pediatric patient presented with microscopic hematuria several days prior to admission, which was believed to be an innocuous finding warranting outpatient follow-up.

This case presents an important reminder that all symptoms, physical exam findings and laboratory data must be utilized in the evaluation and management of patients, even those that appear to be insignificant. Taken together, the sum of the pieces may represent a different clinical picture than each piece evaluated separately. The disease impact of SARSCoV-2 is such that even when patients recover from the acute physiologic derangements associated with this virus, extensive rehabilitation services may be necessary in order to return the patient to baseline functioning.

\section{Acknowledgments}

The authors wish to acknowledge Ms. Wanda Thomas and Ms. Detra Walton at the Mercer University Skelton Medical Library for their efforts in securing reference materials.

\section{Financial Disclosure}

None to declare.

\section{Conflict of Interest}

Neither Dr. Gordon nor Dr. Dinerman has any conflict of interest to report.

\section{Informed Consent}

None to declare.

\section{Author Contributions}

Dr. Gordon researched, drafted and edited the report. Dr. Dinerman edited and provided guidance and mentorship in the drafting of the report.

\section{Data Availability}

The authors declare that data supporting the findings of this study are available within the article.

\section{References}

1. WHO, 2020. Report of the WHO-China joint mission on coronavirus disease 2019 (COVID-19). [online] Available at: https:/www.who.int/docs/default-source/coronaviruse/who-china-joint-mission-on-covid-19-final-report. pdf. Accessed March 23, 2020.

2. Coronavirus (COVID-19). United States Centers for disease control and prevention. Available at: https://www. cdc.gov/coronavirus/2019-ncov/index.html (CDC website). Accessed March 24, 2020.

3. Guan WJ, Ni ZY, Hu Y, Liang WH, Ou CQ, He JX, Liu L, et al. Clinical characteristics of coronavirus disease 2019 in China. N Engl J Med. 2020;382(18):1708-1720.

4. European Centre for Disease Prevention and Control. 2020. COVID-19. [online] Available at: https://www.ecdc. europa.eu/en/novel-coronavirus-china. Accessed March 23, 2020.

5. Pan L, Mu M, Yang P, Sun Y, Wang R, Yan J, Li P, et al. Clinical characteristics of COVID-19 patients with digestive symptoms in Hubei, China: a descriptive, cross-sectional, multicenter study. Am J Gastroenterol. 2020;115(5):766-773.

6. Wu Z, McGoogan JM. Characteristics of and important lessons from the coronavirus disease 2019 (COVID-19) outbreak in China: summary of a report of 72314 cases from the Chinese Center for Disease Control and Prevention. JAMA. 2020.

7. Dong Y, Mo X, Hu Y, Qi X, Jiang F, Jiang Z, Tong S. Epidemiology of COVID-19 Among Children in China. Pediatrics. 2020:e20200702.

8. Wan W, Achenbach J. Coronavirus is mysteriously sparing kids and killing the elderly. Understanding why may help defeat the virus. Washington Post. https://www. washingtonpost.com/health/2020/03/10/coronavirus-ismysteriously-sparing-kids-killing-elderly-understandingwhy-may-help-defeat-virus/. Published March 10, 2020. Accessed March 24, 2020.

9. Mehta P, McAuley DF, Brown M, Sanchez E, Tattersall RS, Manson JJ, Hlh Across Speciality Collaboration UK. 
COVID-19: consider cytokine storm syndromes and immunosuppression. Lancet. 2020;395(10229):1033-1034.

10. Ruan Q, Yang K, Wang W, Jiang L, Song J. Clinical predictors of mortality due to COVID-19 based on an analysis of data of 150 patients from Wuhan, China. Intensive Care Med. 2020;46(5):846-848.

11. Lippi G, Plebani M, Henry BM. Thrombocytopenia is associated with severe coronavirus disease 2019 (COVID-19) infections: A meta-analysis. Clin Chim Acta. 2020;506:145-148.

12. Zarychanski R, Houston DS. Assessing thrombocytopenia in the intensive care unit: the past, present, and future. Hematology Am Soc Hematol Educ Program. 2017;2017(1):660-666.

13. Drews RE, Weinberger SE. Thrombocytopenic disorders in critically ill patients. Am J Respir Crit Care Med. 2000;162(2 Pt 1):347-351.

14. Shi H, Han X, Jiang N, Cao Y, Alwalid O, Gu J, Fan Y, et al. Radiological findings from 81 patients with COVID-19 pneumonia in Wuhan, China: a descriptive study. Lancet Infect Dis. 2020;20(4):425-434.

15. Bernheim A, Mei X, Huang M, Yang Y, Fayad ZA, Zhang $\mathrm{N}$, Diao K, et al. Chest CT findings in coronavirus disease-19 (COVID-19): relationship to duration of infection. Radiology. 2020:200463.

16. Nn M, Lee E, Yang J, Yang F, Li X, Wang H, Lui MM, et al. Imaging profile of the COVID-19 infection: radiologic findings and literature review. Radiology: Cardiothoracic Imaging. 2020;2(1):13.

17. Cheng Y, Luo R, Wang K, Zhang M, Wang Z, Dong L, Li $\mathrm{J}$, et al. Kidney impairment is associated with in-hospital death of COVID-19 patients. MedRxIV.

18. Davis R, Jones JS, Barocas DA, Castle EP, Lang EK, Leveillee RJ, Messing EM, et al. Diagnosis, evaluation and follow-up of asymptomatic microhematuria (AMH) in adults: AUA guideline. J Urol. 2012;188(6 Suppl):2473-2481. 